\title{
Niveles de desarrollo de la competencia digital docente: una mirada a marcos recientes del ámbito internacional
}

\section{Proficiency levels of teachers' digital competence: a review of recent international frameworks}

\author{
Angelina Lorelí Padilla-Hernández ${ }^{1}$, Vanesa M. ${ }^{a}$ Gámiz-Sánchez², \\ M. ${ }^{a}$ Asunción Romero-López ${ }^{3}$ \\ ${ }^{1}$ Universidad de Granada, España (loreli@correo.ugr.es) \\ ${ }^{2}$ Departamento de Didáctica y Organización Escolar, Universidad de Granada, España (vanesa@ugr.es) \\ ${ }^{3}$ Departamento de Didáctica y Organización Escolar, Universidad de Granada, España (romerol@ugr.es)
}

Recibido el 9 de febrero de 2019; revisado el 1 de abril de 2019; aceptado el 3 de abril de 2019; publicado el 1 de diciembre de 2019

\section{RESUMEN:}

El desarrollo de la competencia digital docente (CDD) es una meta educativa que ha sido reconocida en el ámbito de la política educativa a partir de marcos y modelos de alcance internacional. En este artículo se abordan tres marcos de CDD que fueron publicados entre 2016 y 2017. El objetivo es analizar los aportes de dichos marcos a la comprensión del desarrollo de la CDD como parte del debate conceptual sobre esta competencia. El método seguido constó de una primera fase de revisión documental, y después se prosiguió con el análisis de contenido de enfoque cualitativo, descriptivo y comparativo. Dos fueron las variables de estudio: niveles de competencia y aspectos para favorecer su desarrollo. En cuanto a los hallazgos de la primera variable, los marcos presentaron tres sentidos de profundización de la CDD: la creación de ambientes de aprendizaje, la mejora de la práctica educativa y la difusión de experiencias de aprovechamiento de las tecnologías de información y comunicación (TIC). Los aspectos que favorecen la profundización de la CDD son: la reflexión, la experiencia continuada, la colaboración y la participación del profesorado en el ámbito de la educación y las TIC. Finalmente, se indican varios desafíos del enfoque de los marcos y de su puesta en práctica, así como cuestiones críticas del apoyo institucional a los docentes a fin de promover el desarrollo de la CDD.
PALABRAS
CLAVE:
COMPETENCIAS DOCENTES, TIC, INTEGRACIÓN DE LA TECNOLOGÍA, ESTÁNDARES, POLÍTICA EDUCATIVA, INNOVACIÓN EDUCATIVA.

\begin{abstract}
:
The development of teachers' digital competence is an educational goal that has been recognized in the field of educational policy through international frameworks and models. This article addresses three frameworks for teachers' digital competence that were published between 2016 and 2017. The objective is to analyze the contributions of these frameworks to the understanding of the development of this competence. The method consisted of both documentary research and content analysis at a qualitative, descriptive and comparative approach, focused on two variables: proficiency levels of competence and aspects to foster competence development. The findings of the first variable pointed to three ways in which the teachers' digital competence could improve: the creation of learning environments, the improvement of educational practice, and the spread of experiences of educational use of information and communication technologies (ICT). Regarding the aspects that lead
\end{abstract}


to the development of this competence, the frameworks showed: reflection, continuous practice, collaboration and the participation of teachers in the field of education and ICT. Finally, some challenges for both the approach of the frameworks and their implementation are highlighted, as well as critical issues about the institutional support to teachers in order to promote the development of their digital competence.

\section{KEYWORDS: TEACHER COMPETENCIES, ICT, TECHNOLOGY INTEGRATION, STANDARDS, EDUCATIONAL POLICY, EDUCATIONAL INNOVATION}

\section{INTRODUCCIÓN}

En términos generales, la competencia digital docente (CDD) se refiere a la competencia profesional que los educadores necesitan para aprovechar las tecnologías digitales en su práctica. Este campo es una de las líneas de investigación actuales de la tecnología educativa que, según Gisbert, González y Esteve (2016), presenta avances en su conceptualización y en el desarrollo de instrumentos para su diagnóstico y evaluación.

Así, la delimitación de lo que significa esta competencia y su desarrollo en áreas, dimensiones o indicadores es un tema de debate actual. Para Janssen et al. (2013) uno de los desafíos es la falta de un lenguaje común porque existen múltiples formas de entender la competencia digital, como varios autores han señalado (Ala-Mutka, 2011; Gallardo-Echenique, 2013; Larraz, 2013). Esta pluralidad conceptual podría afectar la toma de decisiones y la práctica relacionadas con el uso de TIC si se traduce en una falta de claridad sobre los términos, su significado y alcance (Janssen et al., 2013).

Desde una perspectiva más amplia, las habilidades y aprendizajes para desenvolverse en contextos digitales se consideran necesarios para toda la ciudadanía y, en consecuencia, uno de los desafíos consiste en la formación del profesorado a fin de promover aprendizajes en esta línea en la educación formal (Atchoarena et al., 2017). Promover la competencia digital en la educación formal se considera un derecho de los estudiantes y una responsabilidad educativa (Tammaro y D'Alessio, 2016). De hecho, el currículum de la formación básica y superior en distintos países comprende la integración de las TIC como un aspecto pertinente para la formación de los estudiantes. En la educación superior esta competencia se entiende como necesaria para la participación en el contexto profesional y social, influenciado por las prácticas y recursos digitales (Area, 2010; Arnao y Gamonal, 2016; Ruiz, 2019).

En consecuencia con lo anterior, la promoción de un perfil docente para el aprovechamiento de los recursos digitales se ha incluido en la política educativa. Muestra de ello son los marcos y modelos del ámbito internacional que han abordado la competencia digital o competencia TIC de los docentes. Uno de dichos marcos es el de competencias y estándares TIC de la Organización de las Naciones Unidas para la Educación, la Ciencia y la Cultura (UNESCO, 2011), que reconoce la importancia del docente para promover el uso de TIC en la enseñanza y el aprendizaje e incluye seis aspectos de su labor: comprensión de las TIC en la educación, currículum y evaluación, pedagogía, TIC, organización y administración y el aprendizaje profesional docente. De acuerdo a este marco se proponen tres niveles de apropiación de las TIC en la educación: alfabetización tecnológica, profundización del conocimiento y creación de conocimiento. Además, se encuentra la línea de trabajo de la Comisión Europea sobre las habilidades y aprendizajes en la era digital, desde la que se han elaborado estudios y caracterizaciones de la competencia digital.

Aparte de su relevancia a nivel de política educativa, los marcos de CDD han impactado en la investigación en tecnología educativa. Algunos estudios suelen partir de este tipo de referentes, además de otros fundamentos teóricos, para delimitar el significado de competencia digital que pretenden diagnosticar o evaluar (Cabero, Llorente y Marín, 2010; Carrera y Coiduras, 2012; LázaroCantabrana, Usart-Rodríguez y Gisbert-Cervera, 2019; Pozos, 2015; Rangel, 2016; Tourón et al., 2018). Inclusive, una vertiente de investigación ha sido el análisis propio de estos marcos a nivel conceptual y de sus implicaciones para la práctica (Castañeda, Esteve y Adell, 2018; Durán, Gutiérrez y Prendes, 2016).

En esta línea destacamos tres puntos de debate. El primero de ellos consiste en las deficiencias del enfoque de los marcos de CDD, como señalan Castañeda et al. (2018). En resumen, los autores indican: la falta de comprensión del rol docente desde una perspectiva holística más allá de centrarse en su acción en el aula, el dejar de lado el contexto sociocultural en donde se pone en juego la competencia, y la visión instrumental de la tecnología como un espacio neutral o que indefectiblemente traerá beneficios a la educación. 
Esta perspectiva propone comprender la CDD de una manera holística al subrayar el carácter integral de la docencia y el compromiso social en el uso de la tecnología (Castañeda et al., 2018).

En segundo lugar, se ha cuestionado la adecuación de un solo marco para cualquier realidad educativa y perfil docente. Es el caso de la exploración de un modelo que explique la competencia TIC de docentes en todos los niveles educativos, desarrollado en el contexto español por Almerich, Orellana, Suárez-Rodríguez y DíazGarcía (2016). Asimismo, diferentes aproximaciones al perfil docente han resaltado variables contextuales (aula, campo disciplinar, escenarios de aprendizaje e institución) como parte de un enfoque competencial de la integración de TIC (Cejas, Navío y Barroso, 2016), así como su impacto en la percepción de bienestar de los docentes que realizan prácticas innovadoras con apoyo de TIC (Valverde, Fernández y Revuelta, 2013). Es posible identificar una tensión entre el nivel de generalidad de las propuestas y el carácter contextual de la propia competencia, como ya indicaba Ala-Mutka (2011) en su revisión conceptual del término competencia digital.

En tercer lugar, la aproximación a la competencia digital conlleva ciertas mediaciones para orientar su desarrollo en la práctica. Esto se puede observar en el diseño de niveles de desarrollo competencial de los marcos y modelos; este interés lleva implícita una visión de la competencia como gradual. En el análisis realizado por Ferrari (2012) de 15 modelos de competencia digital se identificaron tres criterios para los niveles competenciales: la edad de los aprendices, la amplitud o profundidad de prácticas con TIC, por ejemplo, en la variedad de herramientas y estrategias digitales empleadas $\mathrm{y}$, por último, el grado de complejidad cognitiva.

Por otra parte, los hallazgos de Janssen et al. (2013) en su consulta a expertos revelaron el carácter dinámico de esta competencia, así como la importancia de la metacognición y las actitudes para su desarrollo. Esto coincide con la propuesta de actitudes en los marcos y modelos de CDD, como el "Marco común de competencia digital docente" desarrollado por el Instituto Nacional de Tecnologías Educativas y Formación del Profesorado (INTEF, 2017) en España.

En síntesis, se hace patente que existen múltiples acercamientos a la comprensión de esta competencia para su orientación en la práctica. Los modelos parten de una propuesta ideal de competencia y, por tanto, presentan un carácter sistémico o funcional, según la clasificación de competencia propuesta por Díaz-Barriga (2011). Si los marcos ofrecen otros descriptores de la competencia pueden aportar elementos para su aplicación pedagógico-didáctica, al desglosarla en aspectos más concretos de la práctica educativa, o para comprenderla desde el enfoque socio-cultural, como un proceso cualitativo y contextual.

Sin embargo, para que esos marcos y modelos cumplan con el objetivo de impactar en la práctica educativa falta profundizar tanto en las implicaciones de su propuesta como en la aplicación de sus orientaciones. En otras palabras, resulta necesario analizar su enfoque y la interacción con docentes, instituciones y realidades educativas. Lo anterior supone que los docentes y la comunidad educativa conocen estos marcos sobre la competencia digital en educación.

Sobre este punto existen estudios que identificaron en el ámbito de la educación superior un desconocimiento generalizado de estos marcos, de las buenas prácticas y la investigación sobre el uso educativo de TIC (Agreda, Hinojo-Lucena y Raso, 2015; Prendes y Gutiérrez, 2013). Por otra parte, las necesidades formativas del profesorado en este nivel educativo apuntaron al área pedagógica como una de las más apremiantes y a la necesidad de promover proyectos de innovación y colaboración en este ámbito (Pozos, 2015). Si este desconocimiento se presentase en docentes de otros niveles educativos, resultaría difícil valorar la propuesta de estos marcos, es decir, si resultan significativos para definir la labor pedagógica con TIC y para identificar áreas de mejora.

A partir de este contexto general, el presente estudio tiene como objetivo analizar los aportes de marcos de alcance internacional y de reciente publicación, con especial énfasis en la comprensión procesual de la CDD. Este aspecto ha sido menos abordado en la literatura según nuestra revisión bibliográfica, y constituye el interés más general del proyecto de investigación sobre la CDD en educación superior al que se adscribe este trabajo. A continuación, se describe el método seguido para esta revisión.

\section{MÉTODO}

Como ya se mencionó, este estudio forma parte de una investigación más amplia enfocada en el carácter evolutivo de la CDD. La búsqueda de literatura especializada en las principales bases de datos académicas (Web of Science, ERIC, Scopus, EBSCO, IRESIE y Redalyc) con descriptores en castellano y en inglés arrojó varios temas de interés, entre ellos el de los modelos y marcos sobre el rol 
docente en el uso de TIC. Estas propuestas se distinguían por su alcance nacional, internacional o local, es decir, acotado a una institución educativa o proyecto de investigación. La revisión de la literatura confirmó la importancia de este tema en el debate conceptual sobre la CDD puesto que tanto los artículos de investigación como las propuestas teóricas recurrían a uno o varios marcos de CDD como fundamento de su aproximación.

Entonces, se detectó la necesidad de analizar esta categoría general como parte del estado del conocimiento. En un principio, se identificaron en la literatura revisada los marcos relacionados con la CDD y se procedió a revisar este listado en función de la actualización o publicación reciente de marcos internacionales. Por otro lado, el tema de investigación requirió centrarnos en las aportaciones sobre etapas o niveles de desarrollo de la CDD. Como se comentó anteriormente, este aspecto ha sido menos abordado en la literatura e implica una aproximación al concepto de CDD y a las estrategias para fomentar dicha competencia.

Fue así que surgió la necesidad de desarrollar el presente estudio cuyo objetivo fue analizar los aportes a la comprensión del desarrollo de la CDD de marcos internacionales de reciente publicación. La primera fase del estudio comprendió la estrategia de identificación de los marcos a revisar, a partir de criterios de inclusión y de estrategias de búsqueda.

Los siguientes criterios de inclusión fueron la base para elegir los marcos a revisar:

- Actualidad. De reciente publicación o actualización, con especial atención a aquellos marcos difundidos en los últimos dos años desde la fecha en que se realizó la revisión (2016-2018). El propósito fue ampliar la cobertura del análisis de marcos de la literatura identificada.

- Alcance internacional. A fin de completar el rubro de marcos cuya amplitud trasciende una institución o un país, y puede ser referente para estudios de distintos contextos. Se prestó atención especial a América y Europa debido al contexto y el enfoque de la investigación en curso.

- Enfoque en la docencia en general. La docencia en distintos niveles y contextos educativos fue otro de los criterios para complementar la base ya existente de literatura en este nivel general.

- Reconocimiento de su pertinencia. Fue importante que estos marcos tuvieran un aval institucional o en el campo de la tecnología educativa, como una muestra de su aportación al debate general y conceptual sobre la CDD.

Las estrategias de búsqueda incluyeron el rastreo de referencias y de marcos en los artículos previamente localizados, la revisión documental de los materiales, la consulta de sitios en línea relacionados con estos marcos o con la CDD a nivel internacional (organismos gubernamentales, sitios informativos del ámbito de tecnología educativa) y la suscripción a perfiles digitales y canales de difusión relacionados con este tema. En total fueron identificados tres marcos que cubrían los criterios de inclusión, publicados entre 2016 y 2017.

El método de análisis de los marcos de CDD se basó en el análisis de contenido (Bardin, 1996), desde una aproximación cualitativa, descriptiva y comparativa. Dos fueron las variables centrales de análisis, definidas a partir de una primera revisión documental de los textos: los niveles de competencia y los aspectos para favorecer su desarrollo. La síntesis de la comparación de los marcos se realizó mediante una matriz que presenta las dos variables para los tres marcos y un esquema representativo de la segunda variable. Desde la perspectiva cualitativa se informó de los hallazgos de esta indagación, que se comparten en el siguiente apartado.

\section{RESULTADOS}

En la primera sección de resultados presentamos los tres marcos analizados a partir de su contextualización, descripción general y de su propuesta en torno a las variables de análisis. La segunda sección ofrece una visión general y comparativa entre los marcos centrada en los niveles de desarrollo de la CDD y los aspectos que favorecen esta progresión.

\subsection{Marcos de CDD: descripción general y abordaje del desarrollo competencial}

\subsubsection{Competencias TIC desde el enfoque pedagógico: marco avalado por la UNESCO}

La propuesta "Competencias y estándares TIC desde la dimensión pedagógica: una perspectiva desde los niveles de apropiación de las TIC en la práctica educativa docente" (Valencia-Molina et al., 2016) fue realizada por la Pontificia Universidad Javeriana de Cali, Colombia, y ha sido respaldada por la UNESCO debido a la orientación pedagógica que ofrece tanto para la formación docente como para la integración de tecnologías digitales a la práctica educativa. Su interés es orientar las competencias docentes para el diseño, la implementación y 
evaluación de escenarios educativos con TIC; éstas constituyen las tres competencias TIC del ámbito pedagógico.

El marco presenta el encuadre teórico de su propuesta que incluye, entre otros temas: el rol del profesorado ante el desafío de las TIC en un contexto como la Sociedad de la Información y el Conocimiento, el uso reflexivo de TIC, la educación de calidad y la apropiación de las TIC. En síntesis, la apropiación de tecnologías por parte de los docentes se entiende como un proceso gradual, “(...) un fenómeno complejo y multifactorial" (ValenciaMolina, et al., 2016, p. 11), por medio del cual el profesorado incorpora las tecnologías a su práctica educativa como parte de los sistemas simbólicos y de los objetivos educativos planteados. El carácter dinámico de este proceso se debe a la influencia de múltiples factores como el conocimiento del docente, el perfil de los estudiantes, el campo de conocimiento y la tecnología. Se afirma que la reflexión del docente sobre el proceso y la eficiencia de las prácticas, su experiencia y la práctica continuada, así como una intencionalidad claramente definida para la integración de TIC son de ayuda para que el docente avance en esta competencia. El aprendizaje significativo y la formación integral de los estudiantes requieren que los docentes desarrollen competencias TIC.

Cada una de las subcompetencias (diseño, implementación y evaluación) se presenta de manera detallada a partir de estándares por nivel de desarrollo, descriptores y situaciones de aplicación. Este detalle se ofrece para el diagnóstico de la práctica docente y para la orientación de la formación y la mejora de la práctica educativa.

Tres son los niveles de desarrollo marcados: integración, reorientación y evolución. En estos niveles se aprecia una progresión que va desde el uso de tecnologías para ahorrar tiempo y facilitar el compartir información y presentar contenidos, hacia la participación activa de los estudiantes en la construcción de conocimiento para llegar a la creación de ambientes flexibles y enriquecidos donde las tecnologías son aprovechadas por su potencial de mediación, y gracias a la colaboración entre docentes. No obstante, el mismo marco propone trascender el rasgo jerárquico de los niveles puesto que una práctica o un docente podrían presentar varios niveles, es decir, no son mutuamente excluyentes ni el avance es siempre lineal o progresivo.

A su vez, cada nivel presenta tres elementos distintos: conocer, utilizar y transformar, que van desde un conocimiento básico de las tecnologías y sus ventajas para la práctica educativa a una propuesta para la creación de escenarios de aprendizaje y estrategias para la evaluación. Finalmente, se llegaría a transformar estos procesos debido a la adaptación de las propuestas educativas, según la valoración del profesorado, de los estudiantes y de otros profesores.

Para procurar el desarrollo de la competencia TIC se incluyen recursos de apoyo como un ejemplo de evaluación de prácticas con TIC y un itinerario formativo para los docentes. Éste último plantea la recuperación de la práctica educativa desde una perspectiva cualitativa, el desarrollo de comunidades de aprendizaje y la sistematización de buenas prácticas.

\subsubsection{La competencia digital de los educadores: marco de la Comisión Europea}

El "Marco europeo para la competencia digital de los educadores", ("DigCompEdu" por sus siglas en inglés), fue propuesto por el Joint Research Centre de la Comisión Europea en 2017 (Redecker, 2017). Tiene como propósito ofrecer una orientación y un lenguaje común para abordar esta competencia, y está dirigido a los diseñadores de políticas educativas, a los educadores, las instituciones y proyectos de los ámbitos formal y no formal. Su diseño implicó la revisión de propuestas y experiencias a nivel internacional, así como una consulta amplia a docentes y expertos del ámbito. Además, este marco forma parte de un proyecto de la Comisión Europea más amplio sobre los aprendizajes y habilidades para la era digital.

Entre los puntos de partida de dicho marco destacamos tres aspectos. El primero de ellos es la figura del docente como un modelo para los estudiantes, por lo que este requiere poseer las competencias que todo ciudadano necesita para participar en una sociedad digital. El segundo es la concepción del docente como facilitador del aprendizaje y, en consecuencia, la importancia de las competencias pedagógicas de su perfil. El tercer aspecto es la relación de la competencia digital con otras áreas competenciales docentes: las profesionales, pedagógicas y del área disciplinar. Adicionalmente, se relaciona la CDD con la competencia digital de los estudiantes, necesaria para su participación en el contexto actual.

Un total de seis áreas y 22 competencias conforman este marco enfocado en los educadores en general. Las seis áreas competenciales son: compromiso profesional, recursos digitales, enseñanza y aprendizaje, evaluación y retroalimentación, empoderar a los estudiantes, y 
facilitar la competencia digital de los estudiantes. Cada competencia es descrita brevemente a partir de una definición $\mathrm{y}$ un listado de actividades representativas a manera de ejemplo. También se incluye una rúbrica con afirmaciones de desempeño según los seis niveles del desarrollo competencial. Esta descripción pretende servir a los docentes como una guía para identificar su nivel además de las acciones que le permitirán ampliar el aprovechamiento de tecnologías digitales.

La CDD se entiende como un proceso de aprendizaje continuo y dicho proceso se contempla en el área de compromiso profesional del marco. Se reconocen los planos individual, grupal y colectivo de esta competencia, hasta incluir a la comunidad educativa. El proceso de desarrollo competencial se desglosa en seis niveles como en el ámbito de los idiomas: novato (A1), explorador (A2), integrador (B1), experto (B2), líder (C1) y pionero (C2). Los niveles más avanzados incluyen a los anteriores, excepto el nivel de novato.

Observamos varios aspectos que distinguen la progresión de niveles: tener experiencia en el uso de herramientas, conocer una gama de estrategias digitales, valorar sus aportaciones a la mejora educativa, actuar con confianza, creatividad y criticidad, y finalmente, ser fuente de aliento y ejemplo para otros profesores. Asimismo, el marco considera que para favorecer el tránsito hacia niveles más avanzados es necesario que los docentes reflexionen, analicen la pertinencia de las estrategias digitales y las diversifiquen, sean críticos con las propuestas propias y de otros, y las renueven.

Como material adicional se presenta un glosario de términos para clarificar conceptos tanto del ámbito educativo como del tecnológico. Por otra parte, se invita a adaptar la propuesta a los diversos contextos de la práctica docente y a las realidades educativas locales y regionales.

\subsubsection{Estándares en TIC para docentes: propuesta del ámbito norteamericano}

Los estándares propuestos por el International Society for Technology in Education (ISTE, 2017) han sido actualizados recientemente, la versión anterior se produjo en 2008. Para realizar esta actualización se consultó a expertos y se invitó a la comunidad educativa mundial a participar de manera abierta en la propuesta de contenidos para los estándares. Esta organización del contexto estadounidense ha desarrollado proyectos de formación, materiales, eventos presenciales $\mathrm{y}$ diversas propuestas entre las que destacan los estándares TIC para docentes, estudiantes y otros agentes educativos.

En la propuesta de estándares de 2017, los docentes son considerados como profesionales empoderados, ciudadanos y catalizadores del aprendizaje. Siete son los roles que agrupan los estándares TIC para el profesorado: aprendiz, líder, ciudadano, colaborador, diseñador, facilitador $\mathrm{y}$ analista. Es importante destacar que se menciona que el docente ayudará a que los aprendices alcancen los estándares TIC para estudiantes.

Cada rol a su vez presenta entre tres y cuatro estándares o indicadores que concretan la acción docente. Mientras que la descripción del rol es de carácter general y puede no incluir una mención explícita al uso de tecnologías, los indicadores apuntan el enfoque de uso de los recursos digitales para la práctica del docente, el aprendizaje de los estudiantes y el impacto en la comunidad educativa en general.

Aunque esta propuesta no incluye niveles de logro de los estándares, se contempla como necesario que el profesorado aprenda y reflexione de manera continua acerca del aprendizaje de los estudiantes, las posibilidades de la tecnología digital y de las propuestas pedagógicas, se desarrolle profesionalmente y aporte al conocimiento de este ámbito. Acerca de los aspectos que ayudan al logro de los estándares, uno de los materiales adicionales utiliza la metáfora del yoga para describir el proceso de aprendizaje del docente (ISTE, n.d.): comenzar por la situación presente del profesorado, estar dispuesto a comprometerse con el proceso, aprender y colaborar con otras personas, y abrirse a nuevas posibilidades de innovación a partir de la confianza en sí mismo, la creatividad y la diversidad de perspectivas.

\subsection{Síntesis comparativa de niveles y aspectos que favorecen el desarrollo de la CDD}

Los tres marcos presentan coincidencias y matices en la forma de entender el desarrollo de la CDD. Vamos a analizar la primera de las variables con la tabla 1 en la que detallamos las diferencias encontradas entre los distintos niveles de desarrollo de la CDD. 
Tabla 1. Niveles de CDD y diferencias en su progresión

\begin{tabular}{|c|c|c|}
\hline Marco & $\begin{array}{l}\text { Niveles de } \\
\text { competencia }\end{array}$ & $\begin{array}{c}\text { Diferencias entre } \\
\text { niveles }\end{array}$ \\
\hline Competencia & Integración & Del uso básico de TIC \\
\hline $\begin{array}{l}\text { TIC: enfoque } \\
\text { pedagógico } \\
\text { (Valencia- }\end{array}$ & $\begin{array}{l}\text { Reorientación } \\
\text { Evolución }\end{array}$ & $\begin{array}{l}\text { hacia la interacción, } \\
\text { construcción de } \\
\text { conocimiento y }\end{array}$ \\
\hline $\begin{array}{l}\text { Molina et al., } \\
\text { 2016) }\end{array}$ & $\begin{array}{c}\text { Elementos: } \\
\text { conocer, utilizar } \\
\text { y transformar. }\end{array}$ & $\begin{array}{l}\text { creación de } \\
\text { información. } \\
\text { Creación de } \\
\text { ambientes de } \\
\text { aprendizaje y } \\
\text { participación de la } \\
\text { comunidad educativa. }\end{array}$ \\
\hline $\begin{array}{l}\text { DigCompEdu } \\
\text { (Redecker, } \\
\text { 2017) }\end{array}$ & $\begin{array}{c}\text { A1. Novato } \\
\text { A2. Explorador } \\
\text { B1. Integrador } \\
\text { B2. Experto } \\
\text { C1. Líder } \\
\text { C2. Pionero }\end{array}$ & $\begin{array}{l}\text { De usos dispersos de } \\
\text { TIC hacia la } \\
\text { experimentación, } \\
\text { ampliación de la } \\
\text { gama de estrategias, } \\
\text { mayor confianza y } \\
\text { creatividad, reflexión } \\
\text { y análisis sobre las } \\
\text { propuestas propias y } \\
\text { de otros. } \\
\text { Eficacia, ser fuente de } \\
\text { inspiración, mejora y } \\
\text { evaluación del uso } \\
\text { pedagógico de TIC. }\end{array}$ \\
\hline $\begin{array}{c}\text { ISTE } \\
\text { Estándares } \\
\text { para docentes } \\
\text { (ISTE, 2017) }\end{array}$ & $\begin{array}{l}\text { No hay niveles } \\
\text { sino roles: } \\
\text { Aprendiz, líder, } \\
\text { ciudadano, } \\
\text { colaborador, } \\
\text { diseñador, } \\
\text { facilitador y } \\
\text { analista. }\end{array}$ & $\begin{array}{l}\text { Los indicadores } \\
\text { implican: } \\
\text { explorar y aplicar } \\
\text { enfoques educativos } \\
\text { con TIC, reflexión } \\
\text { continua sobre la } \\
\text { práctica, participar en } \\
\text { redes, ser ejemplo y } \\
\text { modelo para los } \\
\text { estudiantes. } \\
\text { Dialogar con la } \\
\text { comunidad educativa } \\
\text { y los interesados en el } \\
\text { ámbito. }\end{array}$ \\
\hline
\end{tabular}

A partir del análisis de la primera variable encontramos que los marcos coinciden en señalar tres sentidos en el aprovechamiento de las TIC por parte del docente: 1) la creación de ambientes de aprendizaje participativos que promuevan la autonomía, la colaboración y la competencia digital de los estudiantes, 2) la mejora de la práctica educativa y docente a partir de la reflexión y la evaluación, integrando las sugerencias de estudiantes, docentes y la comunidad educativa y 3 ) compartir el conocimiento generado a partir de la experiencia y ayudar a otros docentes a avanzar en el aprovechamiento de TIC. Estos sentidos requieren que los docentes aprendan de manera continua, pongan en práctica estrategias pedagógicas donde las TIC tienen aportes específicos y a la vez son ambientes de interacción, y evalúen de manera continua el proceso y los resultados.

En cuanto a la segunda variable, aspectos que ayudan a favorecer el tránsito de niveles, se identificaron los siguientes puntos:

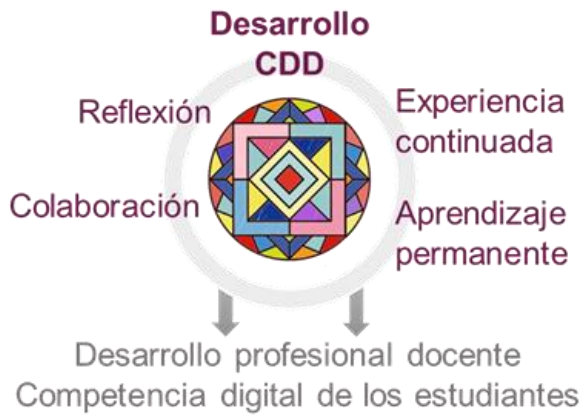

Figura 1. Aspectos que ayudan a favorecer el tránsito de niveles

- La reflexión es clave para los tres modelos y es entendida en general como la valoración de las prácticas en términos de sus resultados, del proceso y de sugerencias para la mejora realizadas por los estudiantes, compañeros y la comunidad educativa. Las TIC son entendidas como una fuente de datos y evidencia sobre el proceso de aprendizaje y las estrategias de enseñanza.

- La experiencia continuada permite a los docentes avanzar en la familiarización con estrategias y recursos digitales. Asimismo, esa continuidad ayuda a evaluar la contribución de las prácticas con TIC al aprendizaje de los estudiantes y al desarrollo de su competencia digital, $\mathrm{y}$ por supuesto, al aprendizaje del profesorado y su desarrollo profesional. Este desarrollo incluye el de los compañeros docentes y el de la comunidad más amplia. Entonces, se apunta hacia un proceso gradual, con rasgos experimentales, aunque con una clara visión de la pertinencia de la integración de las TIC en la educación.

- La colaboración es otro ingrediente fundamental para esta evolución. Por una parte, las propuestas de CDD mencionan la colaboración como enfoque de aprendizaje, y por otra, la colaboración como el diálogo con los estudiantes, compañeros docentes, padres de familia, la comunidad educativa y los interesados 
en el ámbito de la educación y las tecnologías. Se subraya la importancia de que los docentes trabajen con compañeros, compartan sus experiencias e incluyan a la comunidad educativa en esta labor.

- El aprendizaje permanente del docente, con un fuerte énfasis en dos sentidos: la actualización del profesorado sobre la investigación, las buenas prácticas y las propuestas metodológicas del ámbito, y su participación a partir de la generación de conocimiento.

La figura 1 presenta los aspectos clave que aportan al desarrollo de la CDD para promover el desarrollo profesional docente y la competencia digital de los estudiantes. Se ha elegido la metáfora del caleidoscopio puesto que cada propuesta de CDD varía en la organización y mención de los aspectos que la componen.

En cuanto a los matices de cada marco sugerimos que éstos se relacionan con su enfoque conceptual y metodológico. Los dos primeros marcos son más extensos y descriptivos en contraste con los estándares del ISTE, que se enfocan más a desempeños deseados a través de roles del profesorado traducidos en estándares. Además, el marco del ámbito colombiano se centra en la dimensión pedagógica de la CDD desde un enfoque cualitativo, mientras que los otros dos tienen un alcance más general e incluyen de manera explícita áreas como el desarrollo profesional docente, el ejercicio de la ciudadanía y la influencia en la competencia digital de los estudiantes. El marco europeo resalta por su carácter abierto a la adaptación de necesidades específicas, lo que contrasta con el detalle de desempeños y actividades por competencia y nivel.

\section{DISCUSIÓN Y CONCLUSIONES}

Los marcos revisados constituyen un aporte al debate conceptual de la competencia digital docente porque delimitan dicha competencia y reconocen su carácter procesual, ya sea a partir de niveles de competencia o de indicadores y ayudas para favorecer el alcance de niveles más complejos de desempeño. Inferimos a partir de esta revisión el carácter evolutivo de dicha competencia puesto que se concibe como un camino hacia una mayor profundización en el aprovechamiento de las prestaciones culturales y educativas de las TIC en la labor docente. Además, la reciente publicación o actualización de los marcos refleja el interés de la política educativa en esta problemática.

Constatamos que los diferentes marcos resaltan el sentido pedagógico, social y de desarrollo profesional docente de esta competencia en el rol de los educadores. Cabe señalar que el enfoque por competencias y estándares para este rol guarda coherencia con el énfasis de los últimos años en la metodología educativa por competencias para el currículum y, por tanto, para la formación y la evaluación docente.

Para los marcos la dimensión pedagógica es la más importante en la CDD. Esta dimensión incluye: la creación de ambientes de aprendizaje y estrategias de enseñanza, la contribución a que los estudiantes amplíen su competencia digital para aprender y participar en la sociedad, y el desarrollo profesional docente, cuyo aprendizaje permanente, mejora profesional y el aporte institucional pueden apoyarse en el uso de TIC. También se ha reconocido la posibilidad que tienen los docentes de inspirar y ayudar a otros compañeros profesores en su comunidad educativa, y extender esta influencia al participar en el ámbito de la Educación y las Tecnologías. Esta colaboración para la innovación con TIC constituye un reto ante el carácter mayormente individual de las prácticas con TIC (Valverde et al., 2013).

La identificación de niveles de progresión tiene sentido en estos marcos porque ayuda a ubicar el desarrollo competencial del profesorado. En dichos niveles se manifiestan relacionados entre sí dos criterios de complejidad que apuntaba Ferrari (2012). El primero es un conocimiento mayor de herramientas digitales y de estrategias más complejas, como la creación de contenidos. El segundo es una complejidad cognitiva mayor en el enfoque para su utilización y en la reflexión sobre la práctica. El factor edad no se refiere aquí.

$\mathrm{Al}$ repasar los hallazgos de este estudio queremos resaltar algunas coincidencias con el debate más amplio sobre la CDD. En primer lugar, la referencia al área pedagógica y al desarrollo profesional docente coincide con la propuesta del marco de la UNESCO sobre competencias TIC (2011) y con la tendencia general de los estudios sobre la CDD referidos. En segundo lugar, la UNESCO consideró niveles de profundización en la integración de TIC con una progresión hacia la innovación educativa, el liderazgo en el uso de TIC y la creación de conocimiento, en sintonía con las propuestas de la competencia TIC desde la dimensión pedagógica y el marco europeo DigCompEdu. Tercero, estos marcos reconocen el carácter evolutivo, no lineal y 
complejo de la competencia digital, señalado en el trabajo de Janssen et al. (2013).

Además, los marcos han contemplado rasgos de la CDD considerados como importantes por otros estudios. Se ha identificado una vinculación fuerte entre competencias tecnológicas y competencias pedagógicas en la CDD (Almerich et al., 2016, Cejas et al., 2016), relación que está presente cuando los tres marcos indican una familiarización y exploración de los recursos TIC y la relevancia de un enfoque pedagógico y de intenciones didácticas para su aprovechamiento. También se ha reconocido el área pedagógica como distintiva de la CDD, como ya se apuntó, incluyendo además las áreas de competencia digital del docente como ciudadano (Durán et al., 2016). El marco del ISTE subraya que el ejemplo del docente como ciudadano en el ámbito digital influye a los estudiantes y es necesaria la formación en esta línea, en consonancia con la reflexión de Tammaro y D'Alessio (2016) sobre la competencia digital como un derecho y un requisito de la formación de los estudiantes en el contexto actual.

Aunque hemos identificado estas coincidencias, también reconocemos los términos diferentes que los marcos emplean para referirse al rol docente en el uso de TIC: competencias TIC, CDD, y estándares TIC para docentes, y a las TIC en sí mismas: tecnologías digitales, TIC, recursos digitales, tecnologías, etcétera. En esta misma línea, las referencias conceptuales que se ofrecen explícitamente en cada marco son diferentes y más extensas en el caso del marco colombiano y del DigCompEdu. Entonces, por un lado, destacamos la pluralidad de propuestas que confirma el carácter multifacético de la competencia digital (Ala-Mutka, 2011; Gallardo-Echenique, 2013; Janssen et al., 2013) y, por otro, la necesidad de profundizar acerca de la concepción misma del docente, de la educación en relación con las TIC y de la aproximación al término de competencia (Castañeda et al., 2018). Como lo contemplan Janssen et al. (2013), esta diversidad puede interpretarse como un riesgo para la confusión conceptual en el área o como una riqueza hacia un diálogo y la construcción de referentes en el campo.

En particular, y en línea con el planteamiento de Castañeda et al. (2018), encontramos tres desafíos sobre el planteamiento conceptual de los marcos analizados. El primero de ellos consiste en ahondar en la propia concepción de competencias que se aborda brevemente en el marco de competencias TIC desde el enfoque pedagógico y en el DigCompEdu. Las referencias que aparecen tienen elementos de carácter funcional puesto que se describen en el ideal y como parte del perfil de los ciudadanos, sin mayores rasgos del contexto sociocultural, aunque sí del proceso de desarrollo competencial. En este sentido consideramos que existe un avance en los marcos puesto que también refieren elementos de la comprensión de la competencia desde los enfoques pedagógicodidáctico y el socioconstructivista (Díaz-Barriga, 2011). Además, se reconoce como un requisito la adaptación de estos marcos a las necesidades de los contextos específicos. Esto incluiría una concreción, por ejemplo, en los recursos digitales a utilizar, entre ellos los destinados a obtener evidencia y registro para evaluar la práctica educativa, con la consideración de sus implicaciones para el manejo de datos y el trabajo que supondría al profesorado.

El segundo desafío conceptual se refiere a la postura frente al determinismo tecnológico de los marcos, señalado también por Castañeda et al. (2018). Aunque queda pendiente un análisis más profundo en esta línea, consideramos que algunos rasgos de los tres marcos subrayan la importancia de la perspectiva pedagógica y de la valoración de posibilidades y riesgos de las TIC por parte de docentes, estudiantes y de la comunidad educativa. Sin embargo, la aproximación de los marcos a este desafío todavía es difusa.

Desde nuestra perspectiva sumamos un tercer desafío: ahondar en la reflexión del profesorado sobre su práctica, conocimiento profesional y su contexto. Los tres marcos indicaron la reflexión como clave del proceso de apropiación con sentido de las TIC, pero no se puntualiza más sobre su comprensión y mediación. Quizás los desafíos señalados se materializan en la práctica, es decir, en la puesta en marcha de estos marcos u orientaciones.

Consideramos que la propuesta de niveles de desarrollo competencial representa una ventaja para orientar los procesos de los docentes siempre y cuando se dialogue con flexibilidad acerca del contexto particular de los proyectos y experiencias de integración de TIC a la educación, con el perfil docente y las áreas disciplinares específicas, y las características de los niveles educativos. En este sentido, los marcos aportaron también estrategias y recursos para la autoevaluación y la formación docente. Sin embargo, además de reconocer el estado de la CDD es necesario valorar las problemáticas, críticas y preguntas de los docentes acerca del aporte de las prácticas con TIC a la mejora educativa.

Como limitación de este estudio señalamos su alcance mayormente descriptivo, con una línea de 
interpretación centrada en las dos variables de análisis. Por otra parte, las líneas de investigación futuras que identificamos son: el análisis en profundidad de la conceptualización de los marcos, la revisión de experiencias de aplicación de los modelos, las estrategias que favorecen la mejora de la CDD y la competencia digital de los estudiantes, y las oportunidades para dialogar con la política educativa a partir de la experiencia de docentes, estudiantes e instituciones educativas.

Por último, queremos señalar que, aunque el rol del profesorado es fundamental, para la adecuada integración de TIC en la educación se requiere del apoyo institucional. Dicho apoyo incluiría: las oportunidades y estrategias para que los docentes y la comunidad educativa reflexionen sobre la CDD, una cultura organizacional y la colaboración como soporte, la gestión del conocimiento para el diálogo entre las experiencias, los planes institucionales y los marcos generales sobre la CDD, y los factores institucionales como los recursos disponibles y los incentivos para el desarrollo profesional docente.

\section{AGRADECIMIENTOS}

La estancia de investigación cuenta con el apoyo de la Beca para estudios en el extranjero del Consejo Nacional de Ciencia y Tecnología (CONACYT), México.

\section{REFERENCIAS}

Agreda, M., Hinojo-Lucena, M. A., y Raso, F. (2015). A Study on ICT Training Among Faculty Members of Spanish Faculties of Education. The New Educational Review, 42(4), 27-39. doi: 10.15804/tner.2015.42.4.02

Ala-Mutka, K. (2011). Mapping digital competence: towards a conceptual understanding. Sevilla: Joint Research Centre. Recuperado http://ftp.jrc.es/EURdoc/JRC67075_TN.pdf

Almerich, G., Orellana, N., Suárez-Rodríguez, J., y Díaz-García, I. (2016). Teachers' information and communication technology competences: A structural approach. Computers \& Education, 100, 110-125. doi: http://dx.ldoi.org/10.1016/j.compedu.2016.05.002

Area, M. (2010). ¿Por qué formar en competencias informacionales y digitales en la educación superior? Revista de Universidad y Sociedad del Conocimiento (RUSC), 7(2), 2-5.

Arnao, M. O., y Gamonal, C. E. (2016). Lectura y escritura con recursos TIC en Educación Superior. Evaluación de la competencia digital. Innoeduca. International Journal of Technology and Educational Innovation, 2(1), 67-73. doi:10.20548/innoeduca.2016.v2i1.1046

Atchoarena, D., Selwyn, N., Chakroun, B., Miaho, F., West, M., y Coligny, C. (2017). Working Group on Education: digital skills for life and work. Suiza: UNESCO. Recuperado de: http://unesdoc.unesco.org/Ulis/cgi- bin/ulis.pl?catno $=259013 \&$ set $=0059 E 5 F E 44 \_3 \_83 \& g p=$ \&lin $=1 \& 11=\mathrm{s}$

Bardin, L. (1996). Análisis de contenido. Madrid: Ediciones Akal.

Cabero, J., Llorente, M. C., y Marín, V. (2010). Hacia el diseño de un instrumento de diagnóstico de "competencias tecnológicas del profesorado" universitario. Revista Iberoamericana de Educación, 52(7), 1-12.

Carrera, F. X., y Coiduras, J. L. (2012). Identificación de la competencia digital del profesor universitario: un estudio exploratorio en el ámbito de las Ciencias Sociales. REDU. Revista de Docencia Universitaria, 10(2), 273-298.

Castañeda, L., Esteve, F., y Adell, J. (2018). ¿Por qué es necesario repensar la competencia docente para el mundo digital? RED. Revista de Educación a Distancia, (56), 1-20. doi: 10.6018/red/56/6

Cejas, R., Navío, A., y Barroso, J. M. (2016). Las competencias del profesorado universitario desde el modelo TPACK (conocimiento tecnológico y pedagógico del contenido). Píxel-Bit. Revista de Medios y Educación, 49, 105-119.

Díaz-Barriga, A. (2011). Competencias en educación. Corrientes de pensamiento e implicaciones para el currículo y el trabajo en el aula. Revista Iberoamericana de Educación Superior (RIES), 5(2), 3-24.

Durán, M., Gutiérrez, I., y Prendes, M. P. (2016). Análisis de modelos de competencia digital del profesorado universitario. Revista Latinoamericana de Tecnología Educativa (RELATEC), 15(1), 97-114. doi: 10.17398/1695-288X.15.1.97

Ferrari, A. (2012). Digital competence in practice: an analysis of frameworks. Sevilla: Joint Research Centre.

Gallardo-Echenique, E. (2013). Competencia digital: revisión integradora de literatura. Revista de Ciencias de la Educación ACADEMICUS, 1(3), 56-62.

Gisbert, M., González, J., y Esteve, F. (2016). Competencia digital y competencia digital docente: una panorámica sobre el estado de la cuestión. RIITE. Revista Interuniversitaria de Investigación en Tecnología Educativa, $\quad 0, \quad 74-83 . \quad$ doi: http://dx.doi.org/10.6018/riite/2016/257631

Instituto Nacional de Tecnologías Educativas y Formación del Profesorado. (2017). Marco común de competencia digital docente. Recuperado de: http://blog.educalab.es/intef/2016/12/22/marco-comunde-competencia-digital-docente-2017-intef/

International Society for Technology in Education (2017). ISTE Standards for Educators. Disponible en: http://www.iste.org/standards/for-educators

International Society for Technology in Education (n.d.). Stretch your Edtech practice. Breathe into the Standards for Educators. Disponible en: https://info.iste.org/educatorstandards-poster

Janssen, J., Stoyanov, S., Ferrari, A., Punie, Y., Pannekeet, K., y Sloep, P. (2013). Experts' views on digital competence: Commonalities and differences. Computers \& Education, $68, \quad 473-481 . \quad$ doi: 10.1016/j.compedu.2013.06.008

Larraz, V. (2013). La competència digital a la Universitat. (Tesis para obtener el grado de doctora). Universidad de Andorra: Andorra. Disponible en: http://www.tdx.cat/handle/10803/113431

Lázaro-Cantabrana, J., Usart-Rodríguez, M., y Gisbert-Cervera, M. (2019). La evaluación de la competencia digital docente: construcción de un instrumento para medir los conocimientos de futuros docentes. Journal of $\mathrm{New}$ 
Approaches in Educational Research, 8(1), 75-81. doi: 10.7821/naer.2019.1.370

Pozos, K. V. (2015). Evaluación de necesidades de formación continua en competencia digital del profesorado universitario mexicano para la sociedad del conocimiento. (Tesis para obtener el grado de doctora). Universidad Autónoma de Barcelona: España. Disponible en: http://hdl.handle.net/10803/382466

Prendes, M. P., y Gutiérrez, I. (2013). Competencias tecnológicas del profesorado en las universidades españolas. Revista de Educación, 36(1), 196-222. doi: 10.4438/1988-592X-RE-2011-361-140

Rangel, A. (2016). Competencias docentes digitales. Propuesta de un perfil. Píxel-Bit. Revista de Medios y Educación, $46,235-248$.

Redecker, C. (2017). European Framework for the Digital Competence of Educators: DigCompEdu. Sevilla: Joint Research Centre. Disponible en: https://ec.europa.eu/jrc/en/digcompedu

Ruiz, A. (2019). Competencia digital y TIC en interpretación: «renovarse o morir». EDMETIC, Revista de Educación $\begin{array}{llll}\text { Mediática } \quad y \quad \text { TIC, } & 8(1), & 55-71\end{array}$ doi:10.21071/edmetic.v8i1.11062

Tammaro, R., y D'Alessio, A. (2016). Teacher Training and Digital Competence: A Pedagogical Recommendation. International Journal of Digital Literacy and Digital Competence (IJDLDC), 7(2), 1-10. doi:10.4018/IJDLDC.2016040101

Tourón, J., Martín, D., Asencio, N., Pradas, S., e Íñigo, V. (2018). Validación de constructo de un instrumento para medir la competencia digital docente de los profesores (CDD). Revista Española de Pedagogía, 76(269), 25-54.

UNESCO (2011). UNESCO ICT competency framework for teachers. París: UNESCO.

Valencia-Molina, T., Serna-Collazos, A., Ochoa-Angrino, S., Caicedo-Tamayo, A. M., Montes-González, J. A., y Chávez-Vescance, J. D. (2016). Competencias y estándares TIC desde la dimensión pedagógica: una perspectiva desde los niveles de apropiación de las TIC en la práctica educativa docente. Cali: Pontificia Universidad Javeriana. Disponible en: http://repositorio.minedu.gob.pe/handle/123456789/475 7

Valverde, J., Fernández, M. R., y Revuelta, F. I. (2013). El bienestar subjetivo ante las buenas prácticas educativas con TIC: su influencia en profesorado innovador. Educación XX1, 16(1), 255-280. 